\title{
In vitro Resistance Pattern of Selected Antifungal Azoles against Candida albicans Biofilms on Silicone Nasogastric Tube
}

\author{
Derick Erl P. Sumalapao ${ }^{1,2 *}$ (D) , Pauline Bridgette D. Salazar ${ }^{2,3}(\mathbb{D})$, Frederico Martin \\ D. Alegre ${ }^{2,3}$ (D) Nelson R. Villarante ${ }^{4}$ and Nina G. Gloriani ${ }^{5}$
}

${ }^{1}$ Department of Epidemiology and Biostatistics, College of Public Health, University of the Philippines Manila, Manila, Philippines. ${ }^{2}$ Biology Department, College of Science, De La Salle University, Manila, Philippines. ${ }^{3}$ College of Medicine, De La Salle Medical and Health Sciences Institute, Cavite, Philippines. ${ }^{4}$ Department of Physical Sciences and Mathematics, College of Arts and Sciences, University of the Philippines Manila, Manila, Philippines. ${ }^{5}$ Department of Medical Microbiology, College of Public Health, University of the Philippines Manila, Manila, Philippines.

\begin{abstract}
Prolonged use of implanted medical devices has been linked with device-associated infections. Since enteral tube feeding is increasing worldwide, the present study described the morphology of Candida albicans biofilms on the surface of silicone nasogastric tube (NGT) using fluorescence microscopy. With the emergence of multidrug-resistant fungal infections, the present study assessed the susceptibility profile of the biofilms to antifungal azoles namely fluconazole, miconazole, voriconazole, and posaconazole using the standard disc diffusion method. Microscopic studies of $C$. albicans biofilms revealed a complex heterogeneous structure with yeast cells and hyphal elements entrenched within a polysaccharide matrix. Planktonic $C$. albicans cells remained susceptible with posaconazole, fluconazole, miconazole, and voriconazole. However, the fungal biofilms exhibited resistance with miconazole. There was a significant reduction in the zone of inhibition on the 24-h, 48-h, and $72-h$ biofilm formation with posaconazole, voriconazole, and fluconazole, respectively. Kinetic investigation on $C$. albicans biofilm with posaconazole revealed a zero-order kinetic profile $\left(R^{2}=0.9774\right)$ whereas with voriconazole exhibited a first-order kinetic profile $\left(R^{2}=0.9974\right)$. These findings can possibly provide information regarding the resistance of fungal biofilms with antifungal azoles. Demonstration of common biofilm features will extend the findings of this study beyond fungi to polymicrobial infections, as new information and insights will influence several disciplines ranging from environmental microbiology to pharmaceutical drug design intended for biofilm-associated infections.
\end{abstract}

Keywords: Posaconazole, fluconazole, miconazole, voriconazole, feeding tube, kinetic equations.

*Correspondence: dpsumalapao1@up.edu.ph

(Received: 20 September 2019; accepted: 08 November 2019)

Citation: Derick Erl P. Sumalapao, Pauline Bridgette D. Salazar, Frederico Martin D. Alegre, Nelson R. Villarante and Nina G. Gloriani, In vitro Resistance Pattern of Selected Antifungal Azoles against Candida albicans Biofilms on Silicone Nasogastric Tube, J Pure Appl Microbiol., 2019; 13(4):1881-1891. https://doi.org/10.22207/JPAM.13.4.01

(C) The Author(s) 2019. Open Access. This article is distributed under the terms of the Creative Commons Attribution 4.0 International License which permits unrestricted use, sharing, distribution, and reproduction in any medium, provided you give appropriate credit to the original author(s) and the source, provide a link to the Creative Commons license, and indicate if changes were made. 


\section{INTRODUCTION}

Enteral tube feeding is a standard of practice for patients who have functional gastrointestinal tracts but cannot acquire their nutritional needs through normal oral intake (De Legge, 2017). One type of tube that is used for short term enteral tube feeding is the nasogastric tube (NGT). This fine tube is inserted through the nostril of the patient, passes along nasopharynx and esophagus, and finally, into the stomach (Best, 2016). Thus, feeding tubes are usually made of either silicone or polyurethane because they can withstand the gastric acid of the stomach and are able to maintain their position for more than 10-14 d (Dougherty \& Lister, 2015). However, the increasing usage of implanted medical devices also increases device-associated infections, such as biofilm formation of fungal species. Candida is the most common genus of fungi that colonizes medical devices by forming resilient biofilm (Desai et al., 2014). Center for Disease Control recognizes C. albicans as the third most commonly isolated bloodstream pathogen in hospitalized patients where mortality rate is up to $50 \%$ (Tsui et al., 2016). Pathogenesis of $C$. albicans is a consequence of its biofilm formation on host or abiotic surfaces. Silva and colleagues (2017) reported that an estimate of 100,000 deaths annually were caused by biofilm infection. Furthermore, Nobile and Johnson (2015) reported in their study that National Institutes of Health identified biofilm formation of $C$. albicans to be the cause of $80 \%$ of all microbial infections, ranging from superficial mycoses to more serious infections with higher mortality rates. Among the different major classes of antifungal drugs available for treating mucosal or systemic candidiasis, azoles are frequently used because of its availability for oral administration, economical price, and less toxicity (Whaley et al., 2016). The utility of enteral tube feeding for patients with complications such as poor voluntary intake or gut dysfunction is increasing worldwide (Blumenstein et al., 2014). The present study described the morphology of $C$. albicans biofilm on the surface of silicone NGT using fluorescence microscopy. One of the major concerns in fungal infections today is the development of multidrugresistant strains due to broad-spectrum antibiotics (Perlin et al., 2015). Hence, the present study assessed the antifungal activity of fluconazole, miconazole, voriconazole, and posaconazole to $C$. albicans biofilms using the standard disc diffusion method. Moreover, the susceptibility profile of the sessile cells to antifungal azoles was described using different kinetic rate equations. Findings of this study will provide additional information on the biofilm formation of $C$. albicans on silicone NGT with emphasis on its morphological features and susceptibility kinetic profile with the selected antifungal azoles.

\section{MATERIALS AND METHODS}

Organism, Culture Conditions, and Inoculum

Candida albicans (ATCC 14053) isolate

was kindly provided by the Department of Medical Microbiology, College of Public Health, University of the Philippines Manila. The organism was maintained, grown, and subcultured at $37^{\circ} \mathrm{C}$ on Sabouraud dextrose agar (SDA) plates $48 \mathrm{~h}$ prior to use. The inoculum was prepared by placing three distinct $1.5-\mathrm{mm} 48$-h colonies into $10 \mathrm{~mL}$ of sterile distilled water warmed to $37^{\circ} \mathrm{C}$ as modified from Andes et al. (2004). The final inoculum was adjusted to $7.46( \pm 0.07) \log _{10} \mathrm{CFU} / \mathrm{mL}$ using serial dilution and the viable fungal counts of the inoculum were confirmed by plating on SDA. Alternatively, the suspension was vortexed for 15 $s$ and its turbidity was adjusted to have the final stock suspension of 1.0 to $5.0 \times 10^{6}$ cells $/ \mathrm{mL}$. The turbidity was adjusted by adding saline solution or more colonies in order to achieve the turbidity produced by the $0.5 \mathrm{McF}$ arland standard at 530 $\mathrm{nm}$ wavelength (National Committee for Clinical Laboratory Standards (NCCLS), 2004).

\section{Candida albicans Biofilm Formation on Silicone Nasogastric Tube}

A modified method (Hawser \& Douglas, 1994; Chandra et al., 2001) for growing biofilms was employed in this study. Disks with surface area of $0.16 \mathrm{~cm}^{2}(0.2 \mathrm{~cm} \times 0.8 \mathrm{~cm})$ from the silicone NGT (Fr5, Medline ${ }^{\circledR}$, NeoMed, Inc.) were prepared. These disks were placed in glass culture plates for sterilization. Once oven-dried, these strips were individually placed in 96-well tissue culture plates. Standardized cell suspensions $(100 \mu \mathrm{L})$ of the final inoculum was applied to the surface of each disk, supplemented with $50 \mathrm{mM}$ glucose $(200 \mu \mathrm{L})$, and then incubated for up to $72 \mathrm{~h}$ at $37^{\circ} \mathrm{C}$ for the biofilm formation. All experimental setups were carried out in triplicates. 


\section{Microscopic Examination of Candida albicans Biofilms}

Assessment on the morphological structure and appearance of the biofilms on the surface of silicone NGT was performed using fluorescence microscopy. To remove the nonadherent cells, disks were individually submerged in a new 96-well plate containing $0.15 \mathrm{M}$ phosphate buffer solution (PBS, $300 \mu \mathrm{L}$ ) as modified from Chandra et al. (2001). The surfaces of the disks with biofilms were stained for $15 \mathrm{~min}$ with $2 \mathrm{~mL}$ Sypro ${ }^{\circ}$ Ruby (Invitrogen, USA) and were subsequently transferred to microscope slides. The dye was used to stain the fungal cell wall, revealing a dense matrix between the hyphae and biofilm (Steinberg et al., 1996). The stained disks were examined under a fluorescence microscope $\left(\lambda_{\text {max }}\right.$ $=450 \mathrm{~nm}$ ).

Susceptibility Kinetic Profile of Candida albicans Biofilms to Antifungal Azoles

Antifungal susceptibility of $C$. albicans planktonic cells and sessile cells $(24,48$, and $72 \mathrm{~h})$ was monitored using the Clinical and Laboratory Standards Institute (CLSI, 2017) standard disc diffusion method. Following biofilm formation, the disc diffusion method was employed to determine zone of inhibition of the antifungal azoles. A standard test tube was prepared by inoculating three colonies of $C$. albicans in $10 \mathrm{~mL}$ sterile distilled water adjusted to $10^{7}$ cells $/ \mathrm{mL}$. Moreover, the disks were individually submerged in a new 96well plate with $0.15 \mathrm{M} \mathrm{PBS}(300 \mu \mathrm{L})$. Varied number of strips were transferred to a test tube containing $2 \mathrm{~mL}$ of sterile distilled water until similar turbidity was obtained with the established standard test tube and confirmed by standard plating procedure. A sterile cotton swab was dipped in the suspension and streaked in many directions over the entire surface of the Mueller-Hinton agar (MHA) plate supplemented with $2 \%$ glucose and $0.5 \mu \mathrm{g} / \mathrm{mL}$ methylene blue. The inoculated agar was air dried for $30 \mathrm{~min}$. With a pair of forceps, each disc containing antifungal drug was dispensed on the surface of the agar distributed in such a way that their distance from one another is no less than $24 \mathrm{~mm}$ from the center (NCCLS, 2004). The four antifungal discs, fluconazole $(25 \mu \mathrm{g})$ (Oxoid Ltd.), posaconazole ( $5 \mu \mathrm{g})$ (Liofilchem Diagnostic), voriconazole $(1 \mu \mathrm{g})$ (Oxoid Ltd.), and miconazole $(10 \mu \mathrm{g})$ (Liofilchem Diagnostic), were placed in the MHA plates, incubated for $24 \mathrm{~h}$ at $37^{\circ} \mathrm{C}$, and then the zone of inhibition $(\mathrm{mm})$ was measured. The zone of inhibition was measured at the transitional phase wherein apparent decrease in the growth and size of colonies was observed. In addition to the Clinical and Laboratory Standards Institute (CLSI, 2017) guidelines, reference obtained from the Susceptibility Testing of Yeasts (STY, 2011) was also considered in the interpretation of the measured zone of inhibition (Table 1).

\section{Numerical Calculations and Data Analyses}

Quantitative measures were subjected to analysis of variance and made use of Bonferroni test in the post hoc analysis. Several kinetic equations including zero-order, first-order, and second-order models were employed in elucidating the rate mechanisms regarding the susceptibility kinetic profile of $C$. albicans biofilms with the selected antifungal azoles. The parameters of these kinetic equations were estimated employing the linearized forms of the kinetic equations (Table 2) using linear regression analysis (Sumalapao, 2017; Sumalapao, 2018).

Table 1. Clinical and Laboratory Standards Institute (CLSI) and Susceptibility Testing of Yeasts (STY) interpretive guidelines on antifungal susceptibility testing

\begin{tabular}{|c|c|c|c|c|c|}
\hline \multirow[b]{2}{*}{ AntifungalAzole } & \multicolumn{3}{|c|}{ Zone of inhibition $(\mathrm{mm})$ interpretation } & \multirow[b]{2}{*}{ Susceptible } & \multirow[b]{2}{*}{ Reference } \\
\hline & Resistant & Intermediate & $\begin{array}{c}\text { Susceptible-Dose } \\
\text { Dependent }\end{array}$ & & \\
\hline Posaconazole $(5 \mu \mathrm{g})$ & $\leq 13$ & - & $14-16$ & $\geq 17$ & STY, 2011 \\
\hline Fluconazole $(25 \mu \mathrm{g})$ & $\leq 14$ & - & $15-18$ & $\geq 19$ & STY, 2011 \\
\hline 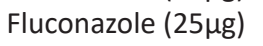 & $\leq 13$ & - & $14-16$ & $\geq 17$ & CLSI, 2017 \\
\hline Miconazole $(10 \mu \mathrm{g})$ & $\leq 11$ & - & $12-19$ & $\geq 20$ & STY, 2011 \\
\hline 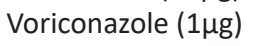 & $\leq 14$ & $15-16$ & - & $\geq 17$ & CLSI, 2017 \\
\hline 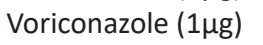 & $\leq 13$ & - & $14-16$ & $\geq 17$ & STY, 2011 \\
\hline
\end{tabular}


Table 2. Nonlinear and linearized forms of the susceptibility kinetic models

\begin{tabular}{|c|c|c|}
\hline Kinetic Model & Nonlinear Model & Linearized Form \\
\hline Zero-order & $\frac{\mathrm{dz}}{\mathrm{dt}}=\kappa_{0}$ & $z_{t}=\kappa_{0} t+z_{0}$ \\
\hline First-order & $\frac{d z_{t}}{d t}=\kappa_{1} z_{t}$ & $\log z_{t}=\log z_{0}+\frac{\kappa_{1}}{2.303} t$ \\
\hline Second-order & $\frac{d z_{t}}{d t}=\kappa_{2} z_{t}^{2}$ & $\frac{1}{z_{t}}=\kappa_{2} t+\left(1 / z_{0}\right)$ \\
\hline
\end{tabular}

\section{RESULTS}

Examination of the biofilm formation via fluorescence microscopy revealed a dense matrix in the areas between the hyphae in a biofilm (Fig. 1). In the initial adherence stage, which occurred during the first $12 \mathrm{~h}$, colonization of $C$. albicans on the surface of the device was observed microscopically. The aggregation complexity increases as the biofilm matures with time. A mature biofilm on the 72-h formation was observed to be embedded in a matrix of exopolysaccharide (Fig. 1C). However, biofilms with less cell density were observed on $72 \mathrm{~h}$ suggesting biofilm detachment and reduction which can lead to possible accumulation of biochemical metabolites and waste products. Microscopic studies of $C$. albicans biofilms on the surface of silicone NGT revealed a complex heterogeneous structure with yeast cells and hyphal elements entrenched within a polysaccharide matrix. Microscopic images revealed a highly heterogeneous architecture of mature $C$. albicans biofilms in terms of the distribution of fungal cells and polysaccharide matrix.
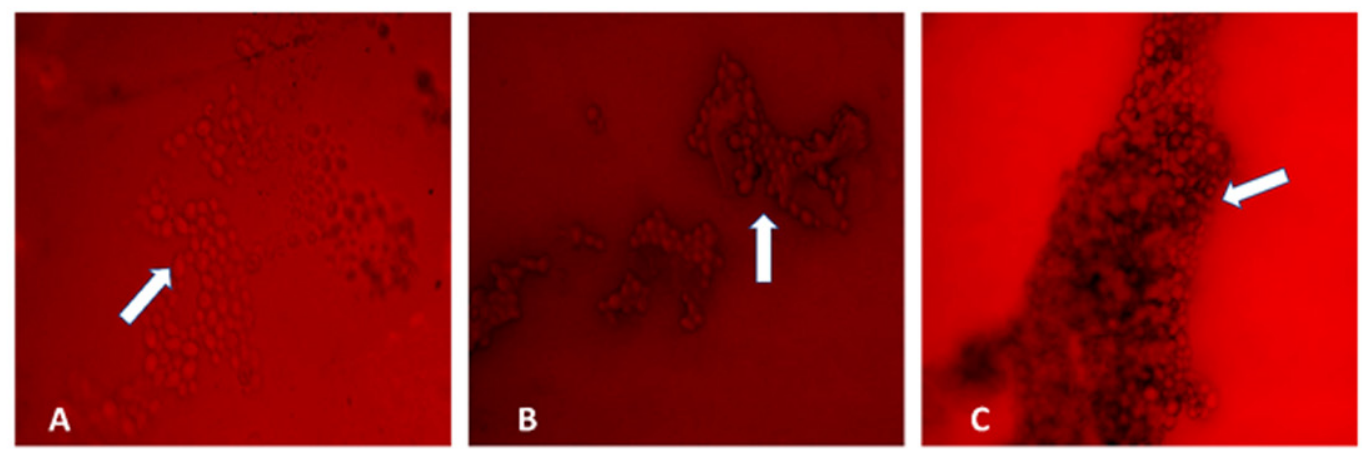

Fig. 1. Fluorescence microscopy of Candida albicans biofilms (in white arrows) on the surface of silicone nasogastric tube at (A) $24 \mathrm{~h},(\mathrm{~B}) 48 \mathrm{~h}$, and (C) $72 \mathrm{~h}$. Magnification: X40

In vitro susceptibility testing of $C$. albicans planktonic cells and biofilms revealed varying profiles with the different antifungal azoles. Some of these representative test plates are presented in Fig. 2 . The planktonic $C$. albicans cells were susceptible to posaconazole, fluconazole, miconazole, and voriconazole (Table 3). However, for the susceptibility profile of fungal biofilms with the antifungal azoles, resistance to miconazole was identified (Table 3). Zone of inhibition with miconazole significantly differed between the planktonic cells and the 24 -h biofilms $(p<0.05)$.
Despite of the resistance profile identified with miconazole, the mean zone of inhibition in the 24-h and 48-h biofilms did not differ (Table 3). However, the 72-h biofilms had a significant reduction in the zone of inhibition when compared with the 24-h biofilms and planktonic cells $(p<0.05)$. Moreover, the fungal biofilms remained susceptible to other antifungal azoles. Although these fungal biofilms were susceptible to fluconazole, a significant reduction in the zone of inhibition on the 72-h biofilm formation was observed (Table 3). Significant reductions in the 


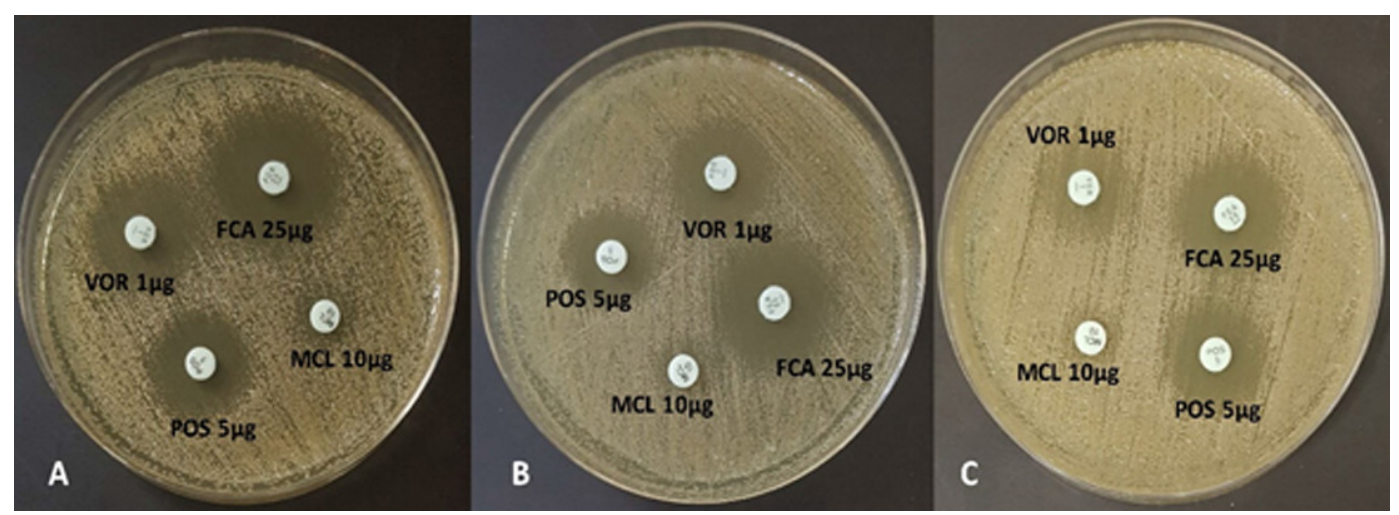

Fig. 2. Susceptibility profile of (A) 24-h, (B) 48-h, and (C) 72-h Candida albicans biofilm: fluconazole (FCA), miconazole $(\mathrm{MCL})$, posaconazole (POS), and voriconazole (VO)

zone of inhibition were also observed as early as $24 \mathrm{~h}$ and $48 \mathrm{~h}$ to posaconazole and voriconazole, respectively (Table 3 ). Zone of inhibition of sessile cells with posaconazole did not differ across the entire biofilm formation $(p>0.05)$. The susceptibility pattern of $C$. albicans biofilms with posaconazole exhibits a zero-order kinetic profile $\left(R^{2}=0.9774\right.$, Table 4$)$ with a mean reduction rate of $0.0972 \mathrm{~mm} / \mathrm{h}$ in the zone of inhibition $(p=0.0114)$. Had resistance pattern of biofilms with voriconazole behaved in the same zeroorder kinetics $\left(R^{2}=0.9955\right)$, there is an average of $0.0830 \mathrm{~mm} / \mathrm{h}$ reduction in the zone of inhibition. However, susceptibility of sessile cells with voriconazole exhibited a first-order kinetic profile $\left(R^{2}=0.9974\right.$, Table 4). The mean reduction in the zone of inhibition is $0.0041 \mathrm{~mm} / \mathrm{h}$ under the firstorder kinetic model ( $p=0.0013$ ) with a significant difference in the susceptibility profile between the 48-h and 72-h biofilm formation (Table 3).

Table 3. Antifungal susceptibility of Candida albicans biofilm on the surface of silicone nasogastric tube to azoles

\begin{tabular}{lcccc}
\hline \multicolumn{4}{c}{$\begin{array}{c}\text { Zone of inhibition } \\
\text { (mean } \pm \text { standard deviation, mm) }\end{array}$} \\
\cline { 2 - 5 } Azoles & Planktonic cells & 24 -hr biofilm & 48 -hr biofilm & 72-hr biofilm \\
\hline Fluconazole $(25 \mu \mathrm{g})$ & $28.33 \pm 0.58^{\mathrm{a}}$ & $25.67 \pm 1.53^{\mathrm{a}}$ & $26.33 \pm 0.58^{\mathrm{a}}$ & $21.33 \pm 1.15^{\mathrm{b}}$ \\
Miconazole $(10 \mu \mathrm{g})$ & $21.33 \pm 0.58^{\mathrm{a}}$ & $11.67 \pm 0.58^{\mathrm{b}}$ & $10.33 \pm 0.58^{\mathrm{bc}}$ & $9.67 \pm 0.58^{\mathrm{c}}$ \\
Posaconazole $(5 \mu \mathrm{g})$ & $24.00 \pm 1.00^{\mathrm{a}}$ & $20.33 \pm 0.58^{\mathrm{b}}$ & $18.67 \pm 0.58^{\mathrm{b}}$ & $18.67 \pm 1.53^{\mathrm{b}}$ \\
Voriconazole $(1 \mu \mathrm{g})$ & $23.67 \pm 0.58^{\mathrm{a}}$ & $21.67 \pm 0.58^{\mathrm{ab}}$ & $21.00 \pm 1.00^{\mathrm{b}}$ & $17.67 \pm 0.58^{\mathrm{c}}$ \\
\hline
\end{tabular}

Values with identical superscript letters in a given row do not differ at $5 \%$ level of significance using Bonferroni test

\section{DISCUSSION}

Candida albicans, a commensal microorganism, can be found on mucosal surfaces such as oral cavity, gut, and vagina (Williams et al., 2013). However, its asymptomatic colonization becomes pathogenic when its local environment is altered because of a change in the immune response of the host. Its development as a pathogenic fungi is caused by its capability to switch morphology and its ability to form biofilm, the latter is considered as an important factor in the contribution of $C$. albicans to human diseases (Tsui et al., 2016). Growth of C. albicans biofilm begins with the formation of basal layer by adhesion of yeast cells on a surface. This basal layer is composed of long tubular cells which can extend to form hyphae, and eventually a mature biofilm can form after addition of proteins and carbohydrates, forming an extracellular matrix (Xu et al., 2019). In the present study, biofilm formation by $C$. albicans initially involved adhesion of yeast cells to the surface of NGT. With the dimorphic switching of the organism from yeast cells to hyphal form, it resulted to matrix formation and complex biofilm 
Table 4. Parameter estimates of the different kinetic rate equations describing the susceptibility of Candida albicans biofilms on the surface of silicone nasogastric tube to antifungal azoles

\begin{tabular}{lccccc}
\hline \multirow{2}{*}{ Kinetic } & Parameter & \multicolumn{3}{c}{ Antifungal Azole } \\
\cline { 3 - 5 } & & Posaconazole & Fluconazole & Miconazole & Voriconazole \\
\hline \multirow{2}{*}{ Zero-order } & $\kappa_{0}$ & -0.0972 & -0.1398 & -0.0655 & -0.0830 \\
& $z_{0}$ & 28.4275 & 17.5836 & 22.4480 & 23.5742 \\
& $R^{2}$ & 0.9774 & 0.5290 & 0.6113 & 0.9955 \\
First-order & $S S E$ & 0.5892 & 36.3623 & 7.3658 & 0.0846 \\
& $p$ & 0.0114 & 0.2306 & 0.2181 & 0.0023 \\
& $\kappa_{1}$ & -0.0040 & -0.0096 & -0.0031 & -0.0041 \\
& $z_{0}$ & 28.5932 & 16.9328 & 22.3590 & 23.7013 \\
& $R^{2}$ & 0.9744 & 0.6398 & 0.6188 & 0.9974 \\
\multirow{5}{*}{ Second-order } & $S S E$ & 0.0002 & 0.0267 & 0.0030 & $2.20 \mathrm{e}-5$ \\
& $\mathrm{p}$ & 0.0129 & 0.2001 & 0.2134 & 0.0013 \\
& $\kappa_{2}$ & 0.0002 & 0.0007 & 0.0001 & 0.0002 \\
& $z_{0}$ & 28.8082 & 16.2800 & 22.2670 & 23.8681 \\
& $R^{2}$ & 0.9688 & 0.6941 & 0.6254 & 0.9954 \\
& $S S E$ & $2.32 \mathrm{e}-6$ & 0.0006 & $3.54 \mathrm{e}-5$ & $5.08 \mathrm{e}-7$ \\
& $\mathrm{p}$ & 0.0157 & 0.1669 & 0.2092 & 0.0023 \\
& & & &
\end{tabular}

$\mathrm{z}_{0}(\mathrm{~mm})$ : zone of inhibition at $\mathrm{t}=0 ; \kappa_{0}(\mathrm{~mm} /$ hour $)$ : zero-order rate constant; $\kappa_{1}(\mathrm{~mm} / \mathrm{hour})$ : first-order rate constant; $\kappa_{2}(\mathrm{~mm}-1$. hour-1): second-order rate constant; R2: coefficient of determination; SSE: sum of squares of the error; $p$ : $p$-value

architectural structure. As a dimorphic organism, C. albicans morphogenetic conversions, the ability to reversibly switch between yeast and filamentous forms, are important for multiple aspects of $C$. albicans biology and pathogenicity. Filamentation seems to play a critical role in the development of the spatially organized architecture observed in mature and highly structured C. albicans biofilm. Hyphae are essential elements for providing structural integrity to fully developed biofilms. When representative microscopic images of the biofilms at varying magnifications were obtained, the thickness of the mature biofilm appears varied over the different areas on the surfaces of the medical device. Individual yeast cells appeared discernible with the less dense biofilm matrix. Moreover, imaging of the biofilms using fluorescence microscopy revealed a heterogeneous structure, a network of extracellular matrix strands, characterized by the presence of yeast cells and hyphal forms. These C. albicans hyphal elements were implicated in fungal infections (Gale et al., 1998; Yaar et al., 1997; Yamada-Okabe et al., 1999).

Fungal infections, device-related biofilm infections caused by $C$. albicans in particular, had been linked with an increase use of medical device among immunocompromised patients or for surgical advances (Shinde et al., 2012). In 2015 , it was reported that these caused $65 \%$ of infections in catheterized patients (Yousif et al., 2015). Recurring infections and death can result from such fungal biofilms (Pandolfi et al., 2019) and these clinical problems are further aggravated by resistance to antifungal drugs. The use of these antifungal drugs depends on the sensitivity profile of species, anatomical site it was found, and the type of infection (De Oliveria Santos et al., 2018). Azoles, which include triazoles and imidazoles, remain to be the largest family of antifungal drugs. Triazoles, which include posaconazole, fluconazole, and voriconazole, are considered in the treatment of superficial and systemic fungal infections (Sheehan et al., 1999). Triazoles inhibit lanosterol 14- $\alpha$-demethylase, an important enzyme in ergosterol biosynthesis pathway, resulting in aberrant sterol intermediates accumulation and subsequent fungal growth restriction (Fera et al., 2009). Similarly, imidazoles such as miconazole respond to induce membrane damage by inhibiting ergosterol synthesis leading to the accumulation of lanosterol. Imidazoles were observed to have fungistatic activity, but a leak in membranes from sterol perturbations has eventually resulted 
in fungal death (Sud \& Feingold, 1981). Azoles inhibit lanosterol 14- $\alpha$-sterol demethylase encoded in ERG11 gene, a gene responsible for ergosterol biosynthesis. Ergosterol is the largest component of fungal cell wall, therefore, its downregulation leads to increased membrane fluidity. Increased membrane fluidity makes the cell vulnerable to toxic $14-\alpha$-methylated sterol that when accumulates inside the cell would result in growth arrest and apoptosis (Musu et al., 2014). In the present study, planktonic $C$. albicans remained susceptible to antifungal azoles, namely posaconazole, fluconazole, miconazole, and voriconazole. Biofilms on the surface of NGT exhibited consistent susceptibility profile to fluconazole. Fluconazole is a broad-spectrum antifungal that is the first choice of drug because of its high efficiency, safety profile, and bioavailability (Liu et al., 2015). Fluconazole, most commonly prescribed antifungal drug for Candida albicans infection, is a triazole antifungal used to treat cryptococcal meningitis in AIDS and oropharyngeal candidiasis. It has been extensively used over the years because of its efficacy as first line drug which is also the principal reason of increasing resistance of $C$. albicans to fluconazole and change of etiological agent of infection from albicans to non-albicans species (Musu et al., 2014). Fluconazole resistance to C. albicans (14.1\%) was reported in 2014 due to repeated use of the drug (Liu et al., 2015). Fluconazole and other triazoles target $C$. albicans by inhibiting cytochrome P450 enzyme lanosterol demethylase in the ergosterol biosynthesis pathway, preventing activation of oxygen and demethylation of lanosterol to become ergosterol. Such inhibition promotes toxic accumulation of methylated sterols in fungal cell which eventually leads to cell death (Berkow \& Lockhart, 2017).

In addition to fluconazole, planktonic cells and biofilms on the surface of NGT also exhibited consistent susceptibility profile to voriconazole. Voriconazole is also an active triazole derived from fluconazole with enhanced antifungal spectrum (Saravolatz et al., 2003). Furthermore, voriconazole and other second-generation azoles were also reported to be fungicidal for some filamentous organisms. Unlike fungistatic drugs, voriconazole completely interrupts ergosterol synthesis resulting to cell death (Saravolatz et al.,
2003). However, a study conducted by Flowers and colleagues (2015) showed that when wildtype azole-susceptible strain of $C$. albicans were exposed to ERG11 alleles carrying an amino acid substitution and tested for susceptibility to voriconazole, substantial decrease in susceptibility was observed. In addition, Wiebusch \& colleagues (2014) tested susceptibility of 24 samples of $C$. albicans against fluconazole and voriconazole. They found out that $54.16 \%$ of $C$. albicans were sensitive to fluconazole while $45.83 \%$ were resistant. A similar result was observed with voriconazole.

Moreover, both the planktonic and sessile cells remained susceptible with posaconazole. Posaconazole is a broad-spectrum triazole used in the treatment of Candida, Aspergillus, and other opportunistic and dimorphic fungi encountered clinically (Gonzalez et al., 2007). Posaconazole is considered a more dominant inhibitor of $14-\alpha$-demethylase sterol and is essentially for prophylaxis of invasive forms of fungal infections (Owotade et al., 2016). In an experiment conducted by Owotade and colleagues (2016), C. albicans strains were collected from the oral cavities of 205 HIV patients, 49 cancer patients, and 20 healthy individuals to test for antifungal susceptibility. Their results showed that seven isolates (3.4\%) of $C$. albicans were resistant to posaconazole. It was also stated in the same study that posaconazole increased its minimum inhibitory concentration from 1.1\% in 2001 to $3.2 \%$ in 2009. Furthermore, Chen and colleagues (2013) demonstrated that posaconazole (0.03$16 \mu \mathrm{g} / \mathrm{mL})$ with caspofungin $(0.25-16 \mu \mathrm{g} / \mathrm{mL})$ exhibited synergistic antifungal activity against $C$. albicans. These cases of increased resistance with posaconazole were presumably caused by a sixth mutation in ERG11, which functions to disrupt the binding segments of the drugs, elevating the level of posaconazole resistance (Li et al., 2015).

The present study identified that the serially grown biofilms were found to be resistant to miconazole. Miconazole was the first azole identified to be effective against systemic infections (Loeffler \& Stevens, 2003). As was described for triazoles, miconazole also functions to affect membrane asymmetry and fluidity, and this altered plasma membrane composition may have contributed to the observed resistance to 
miconazole (Loeffler \& Stevens, 2003). Miconazole is an exception to fungistatic azoles because of its fungicidal effect against yeast cells by accumulating reactive oxygen species (ROS). De Cremer and colleagues (2015) found that miconazole and artemisinins combination have the potential to treat infection caused by biofilm formation of one strain of $C$. albicans. Artemisinins are antimalarial drugs that were found to act synergistically with miconazole because of dihydroartemisinin, a structural homologue of artesunate, a semisynthetic derivative of artemisinin, and its ability to increase the capability of miconazole against biofilms of $C$. albicans (De Cremer et al., 2015). Furthermore, another study conducted by De Cremer and colleagues (2016) also showed that combination of miconazole $(125 \mu \mathrm{M})$ to antimycin A $(25 \mu \mathrm{M})$ exhibited high superoxide induction, which is significant to fungicidal compounds and their production of ROS to increase susceptibility of miconazole to biofilms.

In the present study, $C$. albicans biofilms exhibited a decreasing susceptibility pattern with the tested antifungal azoles. In particular, the susceptibility pattern of $C$. albicans biofilms with posaconazole has a kinetic profile described by a zero-order rate equation. Posaconazole is a new triazole antifungal drug which exhibits a similar mechanism of action as other azole derivatives. Moreover, C. albicans biofilms on the surface of silicone NGT remained susceptible to fluconazole and voriconazole, although a significant reduction in the zones of inhibition was noted as early as 48-h biofilm formation. Nett et al. (2014) established the tolerance to fluconazole of in vitro and in vivo C. albicans biofilms on silicone urinary catheter. Fluconazole was selected primarily for its clinical utility in the treatment of urinary candidiasis since the presence of a halogenated phenyl ring increases its antifungal activity (Dash \& Elmquist, 2001). Fluconazole basically alters the cellular membranes which leads to increased membrane permeability, impaired uptake of precursor, and leakage of essential elements from the cell (Charlier et al., 2006). However, Nasrollahi et al. (2015) reported resistance of C. albicans to fluconazole since Pir1p, a protein responsible for cell wall maintenance, was found overexpressed in the fungal cell wall due to prolonged fluconazole treatment. Moreover, another triazole examined in the present study was voriconazole, a secondgeneration triazole and a synthetic derivative of fluconazole. Candida albicans biofilms when compared to planktonic cells exhibited a significantly different susceptibility pattern, but the fungal biofilm remained susceptible to voriconazole. This might be attributed to the stronger affinity of voriconazole with lanosterol 14- $\alpha$-demethylase which results in complete disruption of ergosterol synthesis and eventual fungal cell death (Saralovatz et al., 2003). Interestingly, the present study identified the resistance of $C$. albicans biofilms on the surface of silicone NGT to miconazole. However, miconazole, as an imidazole, should have its cumulative effect on C. albicans consistent with its direct interaction with the fungal plasma membrane causing growth inhibition, and its contact with the intracellular membranes resulting in cellular disorganization, and eventual fungal death (Cope, 1980).

The planktonic C. albicans is different from $C$. albicans in the biofilms on the surfaces of medical devices. The fungal biofilm exhibited tolerance to some antifungal drugs and biofilms are becoming progressively resistant to the firstline and second-line antifungal drugs (Martin, 1999). Some of the major mechanisms which lead to azole resistance include reduced drug intracellular accumulation, decreased target affinity, and drug effect counteraction (Sanglard, 2016; Spampinato \& Leonardi, 2013). Resistance of biofilms to antifungal drugs is found to be multifactorial, a combination of the $\beta-1,3$ glucan drug sequestration activity, upregulation of several efflux pump genes in the biofilms, and the presence of persister cells (Desai et al., 2014). Several documents alarmingly reported the developing resistance of $C$. albicans to azoles. Mutations in ERG11 have been observed to alter substitution of amino acids, which leads to the revision of mechanisms of azoles, preventing ERG11 inhibition resulting to resistance (Flowers et al., 2015). Another mechanism involved in drug-resistance property of $C$. albicans is the upregulation of several efflux pump genes, which specifically increases resistance of $C$. albicans cell membrane to azoles during early stages of biofilm development. CDR1 and CDR2 ATP binding cassette transporters, along with MDR1, a major facilitator transporter were found in low concentrations in 
clinical isolates without exposure to antifungal resistance (Silva et al., 2017). In increased levels, C. albicans cell membrane also exhibited increased drug resistance to azoles. Furthermore, Silva and colleagues (2017) reported that efflux pumps are time-specific, and higher expression are observed in 12-h biofilm formation, in comparison to a 48-h mature biofilm formation. A group of cells, called persister cells, may also contribute to biofilm drug resistance (Palkova \& Vichova, 2016). In Candida biofilms, persister cells have the capacity to repopulate the biofilm even after they were eliminated by amphotericin B and chlorhexidine (Palkova \& Vichova, 2016). The resistance of these cells can be attributed to the upregulated production of proteins involved in its virulence and stress responses (Li et al, 2015). Due to these emerging cases of antifungal drug resistance, there is an increase in the synthesis and manufacture of new drugs. However, some newer generation azoles have no significant activity against $C$. albicans biofilms (Katragkou et al., 2008).

\section{CONCLUSION}

Candida albicans biofilms on the surface of NGT revealed a complex structure with hyphal elements and yeast cells entrenched within a polysaccharide matrix. These biofilms were resistant to miconazole but remained susceptible to fluconazole and voriconazole, although significant reduction in the zone of inhibition was observed as the biofilm matures. The susceptibility kinetic profile of the fungal biofilm with posaconazole can be described using the zero-order rate kinetic model. In the present study, information on model biofilms with focus on structural organization and susceptibility pattern can possibly provide baseline information which can lead to further resistance mechanism elucidation and subsequent development of novel therapies.

\section{ACKNOWLEDGMENTS}

The organism used in this study was kindly provided by the Department of Medical Microbiology, College of Public Health, University of the Philippines Manila. Fluorescence microscopy was performed in the Molecular Biology Unit Laboratory, De La Salle University, Manila, Philippines.

\section{CONFLICT OF INTEREST}

The authors declare that there is no conflict of interest.

\section{AUTHORS' CONTRIBUTION}

All authors listed have made a substantial, direct, and intellectual contribution to the work, and approved it for publication.

\section{FUNDING}

None.

\section{DATA AVAILABILITY}

All datasets analyzed in the study are included in the manuscript and presented as tables and figures.

\section{ETHICS STATEMENT}

The study protocol was approved by the University of the Philippines Manila Research Ethics Board (UPMREB 2018-486-01). The biosafety clearance was granted by the University of the Philippines Manila Institutional Biosafety and Biosecurity Committee (UPMIBBC 2018-018).

\section{REFERENCES}

1. Andes, D., Nett, J., Oschel, P., Albrecht, R., Marchillo, K. \& Pitula, A. Development and characterization of an in vivo central venous catheter Candida albicans biofilm model. Infection and Immunity, 2004; 72(10): 6023-6031. https://doi.org/10.1128/IAI.72.10.60236031.2004

2. Berkow, E.L. \& Lockhart, S.R. Fluconazole resistance in Candida species: a current perspective. Infection and Drug Resistance, 2017; 10: 237-245. https://doi. org/10.2147/IDR.S118892

3. Best, C. How to insert a nasogastric tube and check gastric position at the bedside. Nursing Standard, 2016; 30(38): 36-40. https://doi.org/10.7748/ ns.30.38.36.s43

4. Blumenstein, I., Shastri, Y.M. \& Stein, J. Gastroenteric tube feeding: techniques, problems and solutions. World Journal of Gastroenterology, 2014; 20(26): 8505-8524. https://doi.org/10.3748/wjg.v20.i26.8505

5. Chandra, J., Kuhn, D.M., Mukherjee, P.K., Hoyer, L.L., McCormick, T. \& Ghannoum, M.A. Biofilm formation by the fungal pathogen Candida albicans: development, structure, and drug resistance. Journal of Bacteriology, 2001; 183(18): 5385-5394. https://doi.org/10.1128/ JB.183.18.5385-5394.2001

6. Charlier, C., Hart, E., Lefort, A., Ribaud, P., Dromer, F., Denning, D. \& Lortholary, O. Fluconazole for the management of invasive candidiasis: where do we stand after 15 years? Journal of Antimicrobial 
Chemotherapy, 2006; 57: 384-410. https://doi. org/10.1093/jac/dki473

7. Chen, Y.L., Lehman, V.N., Averette, A.F., Perfect, J.R. \& Heitman, J. Posaconazole exhibits in vitro and in vivo synergistic antifungal activity with caspofungin or FK506 against Candida albicans. Public Library of Science One, 2013; 8(3). https://doi.org/10.1371/ journal.pone.0057672

8. Clinical and Laboratory Standards Institute. Method for antifungal disk diffusion susceptibility testing of yeasts; approved guideline (Second Edition). PA Wayne, 2017.

9. Cope, J. Mode of action of miconazole on Candida albicans: effect on growth, viability, and $\mathrm{K}^{+}$release. Journal of General Microbiology, 1980; 119: 245-251. https://doi.org/10.1099/00221287-119-1-245

10. Dash, A. \& Elmquist, W. Fluconazole. Analytical Profiles of Drug Substances and Excipients, 2001; 67-113. https://doi.org/10.1016/S1075-6280(01)27005-0

11. De Cremer, K., Lanckacker, E., Cools, T.L., Bax, M., De Brucker, K., Cos, P., Cammue, B.P.A. \& Thevissen, K. Artemisinins, new miconazole potentiators resulting in increased activity against Candida albicans biofilms. Antimicrobial Agents and Chemotherapy, 2015; 59(1): 421-426. https://doi.org/10.1128/AAC.04229-14

12. De Cremer, K., De Brucker, K., Staes, I., Peeters, A., Van den Driessche, F., Coenye, T., Cammue, B.P. \& Thevissen, K. Stimulation of superoxide production increases fungicidal action of miconazole against Candida albicans biofilms. Scientific Report, 2016; 6: 27463. https://doi.org/10.1038/srep27463

13. De Legge, M. Enteral access and associated complications. Gastroenterology Clinics of North America, 2017; 47(1): 23-37. https://doi.org/10.1016/j. gtc. 2017.09.003

14. De Oliveira Santos, G.C., Vasconcelos, C.C., Lopes, A.J.O., de Sousa Cartagenes, M., Do, S., Filho, A.K.D.B., do Nascimento, F.R.F. \& de Andrade Monteiro, C. Candida infections and therapeutic strategies: mechanisms of action for traditional and alternative agents. Frontiers in Microbiology, 2018; 9: 1351. https://doi.org/10.3389/fmicb.2018.01351

15. Desai, J.V., Mitchell, A.P. \& Andes, D.R. Fungal biofilms, drug resistance, and recurrent infection. Cold Spring Harbor Perspectives in Medicine, 2014; 3. https://doi. org/10.1101/cshperspect.a019729

16. Dougherty, L. \& Lister, S. The Royal Marsden Manual of Clinical Nursing Procedures (Ninth edition). Hoboken, NJ; John Wiley \& Sons, 2015.

17. Fera, M., La Camera, E. \& De Sarro, A. New triazoles and echinocandins: mode of action, in vitro activity, and mechanisms of resistance. Expert Review of Anti-Infective Therapy, 2009; 7: 981-998. https://doi. org/10.1586/eri.09.67

18. Flowers, S.A., Colod, B., Whaley, S.G., Schuler, M.A. \& Rogers, P.D. Contribution of clinically derived mutations in ERG11 to azole resistance in Candida albicans. Antimicrobial Agents and Chemotherapy, 2015; 59(1): 450-460. https://doi.org/10.1128/ AAC. 03470-14

19. Gale, C.A., Bendel, C.M., McClellan, M., Hauser, M., Becker, J.M., Berman, J. \& Hostetter, M.K. Linkage of adhesion, filamentous growth, and virulence in
Candida albicans to a single gene, INT1. Science, 1998; 279: 1355-1358. https://doi.org/10.1126/ science.279.5355.1355

20. Gonzalez, G.M., Robledo, E., Saldivar, D., Gonzales, G., Bosques, F. \& Garza, E. Therapeutic efficacy of posaconazole against isolates of Candida albicans with different susceptibilities to fluconazole in a vaginal model. Medical Mycology, 2007; 45(3): 221-224. https://doi.org/10.1080/13693780601164298

21. Hawser, S.P. \& Douglas, L.J. Biofilm formation by Candida species on the surface of catheter materials in vitro. Infection and Immunity, 1994; 62: 915-921.

22. Katragkou, A., Chatzimoschou, A., Simitsopoulou, M., Dalakiouridou, M., Diza-Mataftsi, E., Tsantali, C. \& Roilides, E. Differential activities of newer antifungal agents against Candida albicans and Candida parapsilosis biofilms. Antimicrobial Agents and Chemotherapy, 2008; 52: 357-360. https://doi. org/10.1128/AAC.00856-07

23. Li, P., Seneviratne, C.J., Alpi, E., Vizcaino, J.A. \& Jin, L. Delicate metabolic control and coordinated stress response critically determine antifungal tolerance of Candida albicans biofilm persisters. Antimicrobial Agents and Chemotherapy, 2015; 59(10): 6101-6112. https://doi.org/10.1128/AAC.00543-15

24. Liu, J., Shi, C., Wang, Y., Li, W., Zhao, Y. \& Xiang, M. Mechanisms of azole resistance in Candida albicans clinical isolates from Shanghai, China. Research in Microbiology, 2015; 166(3): 153-161. https://doi. org/10.1016/j.resmic.2015.02.009

25. Loefller, J. \& Stevens, D.A. Antifungal drug resistance. Clinical Infectious Diseases, 2003; 36(1): S31-S41. https://doi.org/10.1086/344658

26. Martin, M.V. The use of fluconazole and itraconazole in the treatment of Candida albicans infections: a review. Journal of Antimicrobial Chemotherapy, 1999; 44: 429-437. https://doi.org/10.1093/jac/44.4.429

27. Musu, M., Evangelista, M., Mura, P., Cossu, A., Carta, M., Aru, G.N. \& Finco, G. Fluconazole therapy for treatment of invasive candidiasis in intensive care patients. Is it still valid from a pharmacological point of view? Journal of Pediatric and Neonatal Individualized Medicine, 2014; 3(1).

28. Nasrollahi, Z., Yadegari, M., Roudbar Mohammadi, S., Roudbari, M., Hosseini Poor, M., Nikoomanesh, F. \& Rajabi Bazl, M. Fluconazole resistance Candida albicans in females with recurrent vaginitis and Pir1 overexpression. Jundishapur Journal of Microbiology, 2015; 8: 1-3. https://doi.org/10.5812/jjm.21468

29. National Committee for Clinical Laboratory Standards (NCCLS). Method for antifungal disk diffusion susceptibility testing of yeasts; approved guideline. 2004 NCCLS document M44-A (ISBN 1-56238-532-1). NCCLS, 940 West Valley Road, Suite 1400, Wayne, Pennsylvania 19087-1898 USA.

30. Nett, J.E., Brooks, E.G., Cabezas-Olcoz, J., Sanchez, H., Zarnowski, R., Marchillo, K. \& Andesa, D.R. Rat in dwelling urinary catheter model of Candida albicans biofilm infection. Infection and Immunity, 2014; 82(12): 4931-4940. https://doi.org/10.1128/ IAI.02284-14

31. Nobile, C.J. \& Johnson, A.D. Candida albicans biofilms 
and human disease. Annual Review of Microbiology, 2015; 69: 71-92. https://doi.org/10.1146/annurevmicro-091014-104330

32. Owotade, F.J., Gulube, Z., Ramla, S. \& Patel, M. Antifungal susceptibility of Candida albicans isolated from the oral cavities of patients with HIV infection and cancer. Journal of the South African Dental Association, 2016; 71(1): 8-11.

33. Palkova, Z. \& Vichova, L. Yeast cell differentiation: lessons from pathogenic and non-pathogenic yeasts. Seminars in Cell \& Developmental Biology, 2016; 57(1): 110-119. https://doi.org/10.1016/j. semcdb.2016.04.006

34. Pandolfi, F., D’Acierno, F., Bortolami, M., De Vita, D., Gallo, F., De Meo, A., Di Santo, R., Costi, R., Simonetti, G. \& Scipione, L. Searching for new agents active against Candida albicans biofilm: a series of indole derivatives, design, synthesis and biological evaluation. European Journal of Medicinal Chemistry, 2019; 165: 93-106. https://doi.org/10.1016/j.ejmech.2019.01.012

35. Perlin, D.S., Shor, E. \& Zhao, Y. Update on antifungal drug resistance. Current Clinical Microbiology Reports, 2015; 2(2): 84-95. https://doi.org/10.1007/s40588015-0015-1

36. Sanglard, D. Emerging threats in antifungal-resistant fungal pathogens. Frontiers in Medicine, 2016; 3(1): 1-10. https://doi.org/10.3389/fmed.2016.00011

37. Saravolatz, L., Johnson, L. \& Kauffman, C. Voriconazole: a new triazole antifungal agent. Clinical Infectious Diseases, 2003; 36: 630-637. https://doi. org $/ 10.1086 / 367933$

38. Sheehan, D., Hitchcock, C., \& Sibley, C. Current and emerging azole antifungal agents. Clinical Microbiology Reviews,1999; 12(1): 40-79. https://doi.org/10.1128/ CMR.12.1.40

39. Shinde, R.B., Raut, J.S. \& Karuppayil, M.S. Biofilm formation by Candida albicans on various prosthetic materials and its fluconazole sensitivity: a kinetic study. Mycoscience, 2012; 53(3): 220-226.

40. Silva, S., Rodrigues, C.F., Araujo, D., Rodrigues, M.E. \& Henriques, M. Candida species biofilms' antifungal resistance. Journal of Fungi, 2017; 3(1): 1-8. https:// doi.org/10.3390/jof3010008

41. Spampinato, C. \& Leonardi, D. Candida infections, causes, targets, and resistance mechanisms: traditional and alternative antifungal agents. Biomed Research International, 2013; 1-13. https://doi. org/10.1155/2013/204237

42. Steinberg, T.H., Haugland, R.P. \& Singer, V.L. Applications of SYPRO orange and SYPRO red protein gel stains. Analytical Biochemistry, 1996; 239: 238-245. https:// doi.org/10.1006/abio.1996.0320
43. Sud, I. \& Feingold, D. Mechanisms of actions of the antimycotic imidazoles. The Journal of Investigative Dermatology, 1981; 76: 438-441. https://doi. org/10.1111/1523-1747.ep12521036

44. Sumalapao, D.E.P. Physiologic kinetic profile of glycemic response in a single dose of clonidine. National Journal of Physiology, Pharmacy and Pharmacology, 2017; 7(7): 701-706.

45. Sumalapao, D.E.P. Growth kinetic profile of Candida albicans under varying environmental conditions. Annual Research and Review in Biology, 2018; 24(6): 1-8. https://doi.org/10.9734/ARRB/2018/40071

46. Susceptibility Testing of Yeasts. Agar diffusion method with Neo-Sensitabs, 2011. http://www.rosco.dk/gfx/ pdf/yeasts.pdf. Accessed 14 May 2018.

47. Tsui, C., Kong, E. \& Jabra-Rizk, M.A. Pathogenesis of Candida albicans biofilm. Pathogens and Diseases, 2016; 74(4): ftw018. https://doi.org/10.1093/femspd/ ftw018

48. Whaley, S.G., Berkow, E.L., Rybak, J.M., Nishimoto, A.T., Barker, K.S. \& Rogers, P.D. Azole antifungal resistance in Candida albicans and emerging non-albicans Candida species. Frontiers in Microbiology, 2016; 7(1): 2173. https://doi.org/10.3389/fmicb.2016.02173

49. Wiebusch, L., Lonchiati, D., Rodrigues, L., Dantas, C., Almeida, A. \& Oliveira, K. Profile susceptibility to fluconazole and voriconazole antifungals by species of Candida albicans isolated from urine culture. BMC Proceeding, 2014; 8(4):34. https://doi. org/10.1186/1753-6561-8-S4-P34

50. Williams, D.W., Jordan, R.P.C., Wei, X.Q., Alves, C.T., Wise, M.P., Wilson, M.J. \& Lewis M.A.O. Interactions of Candida albicans with host epithelial surfaces. Journal of Oral Microbiology, 2013; 5(10): 1-8. https://doi. org/10.3402/jom.v5i0.22434

51. Xu, K., Wang, J.L., Chu, M.P., Jia, C. Activity of coumarin against Candida albicans biofilms. Journal de Mycologie Medicale, 2019; 29(1): 28-34. https:// doi.org/10.1016/j.mycmed.2018.12.003

52. Yaar, L., Mevarech, M. \& Koltin, Y. A Candida albicans RAS-related gene (CaRSR1) is involved in budding, cell morphogenesis and hypha development. Microbiology, 1997; 143: 3033-3044. https://doi. org/10.1099/00221287-143-9-3033

53. Yamada-Okabe, T., Mio, T., Ono, N., Kashima, Y., Matsui, M., Arisawa, M., Yamada-Okabe, H. Roles of three histidine kinase genes in hyphal development and virulence of the pathogenic fungus Candida albicans. Journal of Bacteriology, 1999; 181(23): 7243-7247.

54. Yousif, A., Jamal, M.A. \& Raad, I. Biofilm-based central line-associated bloodstream infections. Advances in Experimental Medicine and Biology, 2015; 830: 157179. https://doi.org/10.1007/978-3-319-11038-7_10 